\title{
A REMARK ON THE NORMALITY OF INFINITE PRODUCTS
}

\author{
KEIKO CHIBA \\ (Communicated by Dennis Burke) \\ Dedicated to Professor Yukihiro Kodama on his 60th birthday
}

\begin{abstract}
In this note we shall prove the following: Suppose that all finite subproducts of a product space $X=\prod_{\beta<\lambda} X_{\beta}$ are normal. If $X$ is $\lambda$ paracompact, then $X$ is normal. Here $\lambda$ stands for an infinite cardinal number.
\end{abstract}

Throughout this note, all spaces are assumed to be Hausdorff and contain at least two points.

Concerning the normality of countable Cartesian products, the following is well known.

(A) (Zenor [5, Theorem A], Nagami [3], or cf. [4, Theorem 6.1]). Suppose that $\prod_{i<n} X_{i}$ is normal for all $n<\omega$. Then a product space $X=\prod_{n<\omega} X_{n}$ is normal iff $X$ is countably paracompact.

Further, Bešlagic proved the following.

(B) (Bešlagić [1, Lemma 3.5]). If a product space $X=\prod_{\beta<\lambda} X_{\beta}$ is normal, then $X$ is $\lambda$-paracompact.

A space $X$ is said to be $\lambda$-paracompact [2] if every open cover of $X$ with cardinality $\leq \lambda$ has a locally finite open refinement.

Let $X=\prod_{\beta<\lambda} X_{\beta}$ be an infinite Cartesian product of spaces. For a finite subset $F$ of $\lambda, \prod_{\beta \in F} X_{\beta}$ is said to be a finite subproduct of $X$.

In this note we shall prove the following.

Theorem. Suppose that all finite subproducts of $X=\prod_{\beta<\lambda} X_{\beta}$ are normal. If $X$ is $\lambda$-paracompact, then $X$ is normal.

Our proof is very similar to the proof of Bešlagić's Theorem 3.4 from [1]. It is also a generalization of Zenor's proof in [5].

First we prove the following lemma.

Lemma. Let $X$ be a $\lambda$-paracompact space and $\mathscr{G}=\left\{G_{\mu} \mid \mu<\lambda\right\}$ be an increasing open cover of $X$. Then there exists an increasing open cover $\mathscr{H}=\left\{H_{\mu} \mid \mu<\lambda\right\}$ of $X$ such that $\mathrm{Cl} H_{\mu} \subset G_{\mu}$ for each $\mu<\lambda$.

Received by the editors December 31, 1986 and, in revised form, March 28, 1988. The author presented the contents of this paper at the 24th Symposium of General Topology on June 2, 1988.

1980 Mathematics Subject Classification (1985 Revision). Primary 54D15, 54B10.

Key words and phrases. Normal, infinite product, $\lambda$-paracompact.

(C) 1989 American Mathematical Society $0002-9939 / 89 \$ 1.00+\$ .25$ per page 
Proof. Since $X$ is $\lambda$-paracompact, there exists a locally finite open cover $\mathscr{V}$ of $X$ which is a refinement of $\mathscr{G}$. Let us put $V_{\mu}=\bigcup\left\{V \mid V \in \mathscr{V}, V \not \subset G_{\alpha}\right.$ for each $\left.\alpha<\mu, V \subset G_{\mu}\right\}$ for each $\mu<\lambda$. Then $\mathscr{V}^{\prime}=\left\{V_{\mu} \mid \mu<\lambda\right\}$ is a locally finite open cover of $X$ such that $V_{\mu} \subset G_{\mu}$ for each $\mu<\lambda$. Let us put $H_{\mu}=X-\mathrm{Cl}\left(\bigcup_{\alpha>\mu} V_{\alpha}\right)$ for each $\mu<\lambda$. Then it is easy to see that $\mathscr{H}=\left\{H_{\mu} \mid \mu<\lambda\right\}$ has the desired properties.

Proof of Theorem. Let us assume that the theorem fails. Then there exist spaces $\left\{X_{\beta} \mid \beta<\lambda\right\}$ such that $X=\prod_{\beta<\lambda} X_{\beta}$ is $\lambda$-paracompact and all finite subproducts of $X$ are normal but $X$ is not normal. Let $\lambda$ be the minimal cardinal number for which such a space exists. Let us prove that " $X$ is normal" which is a contradiction. We shall use the following notation. For each $A \subset \lambda$, let us put $Y_{A}=\prod_{\beta \in A} X_{\beta}$ and let $\pi_{A}: X \rightarrow Y_{A}$ be the projection and for each $\gamma<\lambda$, put $Z_{\gamma}=Y_{\lambda-\gamma}$.

Let $\mathscr{G}=\left\{G_{1}, G_{2}\right\}$ be an arbitrary binary open cover of $X$. We shall show that there exist closed sets $L_{1}$ and $L_{2}$ such that $L_{i} \subset G_{i}$ for $i=1,2$ and $X=L_{1} \cup L_{2}$.

For each $\gamma<\lambda$ and $i=1,2$, let us put

$$
U_{\gamma, i}=\bigcup\left\{U \mid U \text { is open in } Y_{\gamma}, U \times Z_{\gamma} \subset G_{i}\right\} \text {. }
$$

Then $U_{\gamma, i}$ is open in $Y_{\gamma}$ such that $U_{\gamma, i} \times Z_{\gamma} \subset G_{i}$. Let us put $O_{\gamma}=$ $\left(\bigcup_{i=1}^{2} U_{\gamma, i}\right) \times Z_{\gamma}$. Then $\left\{O_{\gamma} \mid \gamma<\lambda\right\}$ is an increasing open cover of $X$. Since $X$ is $\lambda$-paracompact, by Lemma, there exists an increasing open cover $\mathscr{S}=$ $\left\{S_{\gamma} \mid \gamma<\lambda\right\}$ of $X$ such that $\mathrm{Cl} S_{\gamma} \subset O_{\gamma}$ for $\gamma<\lambda$. Let us put $T_{\gamma}=$ $Y_{\gamma}-\pi_{\gamma}\left(X-\mathrm{Cl} S_{\gamma}\right)$ and $C_{\gamma}=\left(\operatorname{Int} T_{\gamma}\right) \times Z_{\gamma}$. Then $T_{\gamma} \subset \bigcup_{i=1}^{2} U_{\gamma, i}$ and

Claim. $\left\{C_{\gamma} \mid \gamma<\lambda\right\}$ is an open cover of $X$.

Proof. It is obvious that $C_{\gamma}$ is open in $X$. Let $x \in X$. Then $x \in S_{\gamma}$ for some $\gamma<\lambda$ and there exist a finite set $F \subset \lambda$ and an open set $U$ in $Y_{F}$ such that $x \in\left(\pi_{F}\right)^{-1}(U) \subset S_{\gamma}$. Let us put $v=\gamma \cup F$. Then $\left(\pi_{F}\right)^{-1}(U) \subset C_{v}$. To show this, let $y=\left(y_{\beta}\right)_{\beta<\lambda} \in\left(\pi_{F}\right)^{-1}(U)-T_{v} \times Z_{v}$. Then $\left(y_{\beta}\right)_{\beta<v} \in \pi_{v}\left(X-\mathrm{Cl} S_{v}\right)$ and so $\left(y_{\beta}\right)_{\beta<v}=\pi_{v}(z)$ for some $z \in X-\mathrm{Cl} S_{v}$. Then $\pi_{F}(z)=\left(y_{\beta}\right)_{\beta \in F} \in U$ because $F \subset v$. Hence $z \in\left(\pi_{F}\right)^{-1}(U)$. However, since $\left(\pi_{F}\right)^{-1}(U) \subset S_{\gamma} \subset S_{v}$, we have $z \in S_{v}$ which is a contradiction.

Since $Y_{\gamma}$ is normal (by the assumption of $\lambda$ ) and $T_{\gamma}$ is closed in $Y_{\gamma}$, there are closed sets $F_{\gamma, 1}$ and $F_{\gamma, 2}$ of $Y_{\gamma}$ such that $F_{\gamma, i} \subset U_{\gamma, i}$ for each $i=1,2$ and $T_{\gamma}=F_{\gamma, 1} \cup F_{\gamma, 2}$. Since $X$ is $\lambda$-paracompact, there is a locally finite open cover $\left\{K_{\gamma} \mid \gamma<\lambda\right\}$ of $X$ such that $K_{\gamma} \subset C_{\gamma}$ for each $\gamma<\lambda$. Let us put $L_{i}=\bigcup\left\{\left(F_{\gamma, i} \times Z_{\gamma}\right) \cap \mathrm{Cl} K_{\gamma} \mid \gamma<\lambda\right\}$ for $i=1,2$. Then $L_{i}$ is closed in $X$ and $L_{i} \subset G_{i}$ for $i=1,2$ and $X=L_{1} \cup L_{2}$. The proof of the theorem is complete.

From this theorem and the previous result (B) of Bešlagic, we obtain the following. This extends the result (A). 
Corollary. Suppose that all finite subproducts of $X=\prod_{\beta<\lambda} X_{\beta}$ are normal. Then $X$ is normal iff $X$ is $\lambda$-paracompact.

Remark. In the theorem, we cannot replace the condition " $X$ is $\lambda$-paracompact" by " $X$ is countably paracompact." In fact, let $X=\prod_{\alpha<\omega_{1}}\left(\omega_{1}\right)_{\alpha}$ where $\left(\omega_{1}\right)_{\alpha}$ is a copy of $\omega_{1}$ with the ordered topology. Then all finite subproducts of $X$ are normal and $X$ is countably paracompact but $X$ is not normal [4, Theorem 6.7].

\section{ACKNOWLEDGMENT}

The author is grateful to the referee for his valuable comments.

\section{REFERENCES}

1. A. Bešlagić, Normality in products, Topology Appl. 22 (1986), 71-88.

2. K. Morita, Paracompactness and product spaces, Fund. Math. 50 (1961), 223-236.

3. K. Nagami, Countable paracompactness of inverse limits and products, Fund. Math. 73 (1972), 261-270.

4. T. C. Przumusiński, Products of normal spaces, Handbook of Set Theoretic Topology (K. Kunen and J. Vaughan, eds.), North-Holland, Amsterdam, 1984.

5. P. Zenor, Countable paracompactness in product spaces, Proc. Amer. Math. Soc. 30 (1971), 199-201.

Department of Mathematics, Faculty of Science, Shizuoka University, Ohya, ShiZUOKA, 422 JAPAN 